\title{
Comprehensive survey of Pc4 and Pc5 band spectral content in Cluster magnetic field data
}

\author{
L. B. N. Clausen and T. K. Yeoman \\ Department of Physics and Astronomy, University of Leicester, Leicester LE1 7RH, UK \\ Received: 22 January 2009 - Revised: 7 August 2009 - Accepted: 12 August 2009 - Published: 19 August 2009
}

\begin{abstract}
In the first part of this study we present two case studies of pulsations that, with the help of ground-based data, are identified as field line resonances (FLRs). These pulsations occurred at frequencies which belong to a set of frequencies that has been suggested to be preferred in the terrestrial magnetosphere (CMS frequencies). We go on to show that for both events there is a significant signature at the same frequency in the time series of the compressional magnetic field observed by the conjugate Cluster satellites. We interpret these as signatures of the compressional mode driving the FLRs. In the second part we present a statistical study including one year's worth of Cluster magnetic field data. For each orbit between May 2004 and June 2005 we identified a three hour interval during which the satellites were located on closed magnetic field lines. We identified peaks in the spectrum between 1.0 and $15.0 \mathrm{mHz}$ of the compressional, poloidal and toroidal components of the magnetic field. We use this database of spectral peaks observed on closed magnetic field lines to investigate whether peaks occur at a preferred set of frequencies which would be indicative for the Earth's magnetosphere behaving like a cavity/waveguide. We find no consistent preference for all CMS frequencies in our dataset, however we do find a preference for certain higher frequencies suggesting that higher harmonics of the cavity/waveguide are a persistent feature of the inner magnetosphere, and are detected by the Cluster spacecraft. This result could be explained by the polar orbit of the Cluster satellites.
\end{abstract}

Keywords. Magnetospheric physics (MHD waves and instabilities; Solar wind-magnetosphere interactions) - Space plasma physics (Waves and instabilities)

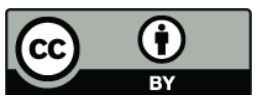

Correspondence to: L. B. N. Clausen (lbnc1@ion.le.ac.uk)

\section{Introduction}

Since ground-based magnetometers were deployed in many regions of the world it is well known that ultra-low frequency (ULF) waves are a ubiquitous feature of the Earth's magnetosphere. As the plasma inside the magnetosphere and indeed the entire solar system is mostly collisionless, they act as the primary agent to transfer mass and energy through the entire system.

Pulsations on the ground were grouped according to their continuous or irregular appearance and their frequency (Jacobs et al., 1964). Whereas the irregular pulsations (termed "Pi") are often connected to the substorm phenomenon (Rostoker et al., 1980), the continuous branch was found to relate to drivers outside the magnetosphere (Takahashi, 1998).

Regular oscillations were termed "Pc" pulsations. One special group of regular pulsations was first interpreted as standing Alfvénic waves on geomagnetic field lines by Dungey (1954). These pulsations are known as field line resonances (FLRs). If measurements of the FLR are available over a range of latitudes, profiles of the signal power at the FLR frequency and the signal's phase can be compiled. In these profiles FLRs are easily identified as resonant features if the power profile shows an about $1^{\circ}$ wide peak at one latitude, and the phase profile exhibits a $180^{\circ}$ phase shift across that resonant latitude (Walker et al., 1979). Generally, the phase propagation is poleward.

Global characteristics like polarization and occurrence distributions of FLRs were first described in detail by Samson et al. (1971) and Samson and Rostoker (1972). Shortly after this, Southwood (1974) and Chen and Hasegawa (1974) were able to explain these characteristics by showing that compressional magnetic waves originating from the magnetopause can couple to Alfvénic waves on closed geomagnetic field lines. They proposed that the compressional waves were generated by surface wave activity originating at the Kelvin-Helmholtz instability (KHI) at both flanks of

Published by Copernicus Publications on behalf of the European Geosciences Union. 
the magnetosphere which thus acts as the primary driver for FLRs.

In the model for the generation of FLRs by Southwood (1974) and Chen and Hasegawa (1974), propagating compressional waves in the near-Earth environment can couple to Alfvénic waves on closed magnetic field lines. As each field line has its own set of eigenfrequencies depending on its length and the mass distribution along that field line, a broad band input of compressional waves should produce a broad band response in the Alfvénic mode, each field line "picking" its resonant frequencies from the broad band input. This has been shown to be the case using spacecraft measurements (Engebretson et al., 1986; Clausen et al., 2009). When looking at ground-based data, however, FLRs rarely show a broad band response.

Instead, monochromatic pulsations were observed on the ground. The frequencies of the pulsations proved to be surprisingly stable with FLRs sometimes resonating over hours with the same frequency, independent of the latitude (i.e. the field line length) at which they were observed.

Kivelson et al. (1984) offered a possible solution to this inconsistency. They proposed, based on observational evidence, that the magnetosphere can for compressional waves be treated as a cavity, bounded by the magnetopause on one side. The other boundary is the frequency dependent position of a turning point of the compressional wave. Between these boundaries a standing compressional wave would form. However, the inner boundary allows energy to leak out of the cavity. The evanescent wave can then, provided a closed field line with a matching eigenfrequency is in the vicinity, couple to an Alfvénic wave on that field line, hence creating a FLR. Naturally, the cavity only supports a discrete set of eigenfrequencies, hence explaining the absence of frequency spreading in ground-based observations of FLRs. This model was subsequently developed further by Kivelson and Southwood (1985) and Kivelson and Southwood (1986).

The cavity model explained the occurrence of stable frequencies which depended on the position of the two boundaries. Later however, several researchers reported not only stable frequencies but a reoccurring stable set of frequencies. Using ground-based magnetometer and HighFrequency (HF) radar data, they found that FLRs tended to occur at frequencies of 1.3, 1.9, 2.6 and $3.4 \mathrm{mHz}$, largely independent of the local time of the observation (Samson et al., 1991; Fenrich et al., 1995; Provan and Yeoman, 1997; Mathie et al., 1999; Plaschke et al., 2009). This set of frequencies is known as "CMS frequencies" - cavity mode model of Samson et al. (1991).

Using the cavity model by Kivelson and Southwood (1985), the predicted eigenfrequencies of the proposed global standing compressional modes were estimated to lie slightly above the CMS frequencies observed on the ground. This inconsistency prompted Samson et al. (1992) to propose that the magnetosphere has to be treated like a waveguide rather than a cavity, therefore allowing azimuthal propaga- tion of the global compressional modes. With this assumption they were able to quite accurately reproduced the observed CMS frequencies.

As FLRs are observed in abundance on the ground, the corresponding driving compressional modes should be observed as often by satellites. This is, however, not the case (Waters et al., 2002). Convincing indirect evidence for the validity of the waveguide model was provided by Samson et al. (1995), however at frequencies significantly higher than the CMS frequencies.

Despite the large body of work that has been done on reoccurring stable frequencies, the topic remains controversial. The most obvious question is how the global mode frequencies can be so stable when the topology of the cavity is so dependent on upstream conditions. The predicted frequencies of both the cavity and the waveguide model depend on the position of the boundaries. Surely the stand-off distance of the magnetopause changes quite significantly, hence changing the properties of the cavity/waveguide. It it thus not obvious why the magnetosphere should still support the same eigenfrequencies while its dimensions are so variable.

The mere preference of FLRs for the CMS frequencies is disputable as well. Baker et al. (2003) analysed pulsation events in ten years of CANOPUS ground-based magnetometer data and while confirming all of the known characteristics of Pc5 wave activity like dawn/dusk asymmetry, they found no preference for any set of frequencies. On the other hand Mathie et al. (1999) found a clear preference for CMS frequencies when analysing 137 pulsation events in IMAGE ground-based magnetometer data. Mathie et al. (1999) however only analysed pulsation events from the month March, "as the equinoctial months have previously been found fruitful for the observation of pulsation events". It may hence be that the preference for CMS frequencies was masked in the study of Baker et al. (2003).

Some researchers have suggested that compressional and Alfvénic MHD oscillations embedded in the solar wind directly drive FLRs in the Earth's magnetosphere (Prikryl et al., 1998). Eriksson et al. (2006) report a number of cases with good correlation between pulsations observed in the solar wind and in HF radar data on the ground. They find the best correlation occurs at frequencies between 0.8 and $1.2 \mathrm{mHz}$. Stephenson and Walker (2002) even present evidence that pressure pulsations in the solar wind drive FLRs on the ground at some of the CMS frequencies.

A recent paper by Viall et al. (2009) summarises work that was done on the occurrence of stable frequencies in groundand space-based measurements. They also show that in about half the cases where a spectral peak was found in solar wind data, the same frequency was statistically significant in a corresponding magnetospheric data segment. However, none of the above cited works is able to explain why pulsations at a certain frequency are more prominent in the solar wind. 


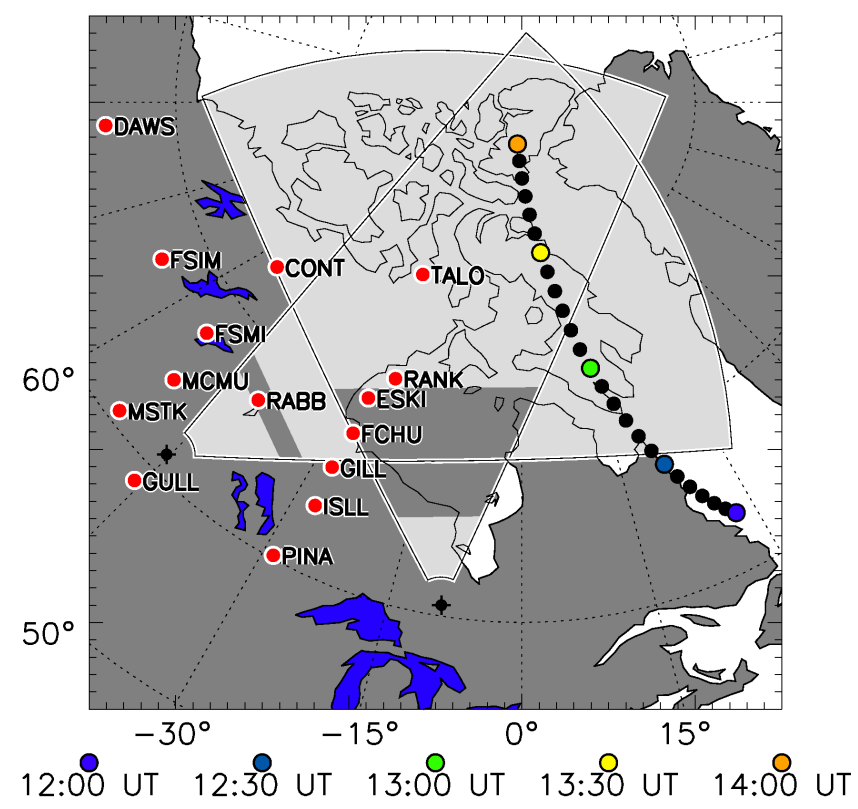

Fig. 1. Locations of the CARISMA magnetometers and the footprint of s/c 1 in geomagnetic coordinates on 24 October 2004. The grey areas give the FOVs of the Saskatoon (looking East) and Kapuskasing (looking North) SuperDARN radar.

\section{Case studies}

In this section we will present two case studies of the 132 intervals that form the statistical study. This aims to emphasise the validity of our statistical results discussed later.

\subsection{October 2004}

Between 12:00 and 14:00 UT on 24 October 2004 the Cluster satellites were magnetically conjugate to the CARISMA array spread over Northern America. The satellite's perigee was located on the dawn flank at $9.1 \mathrm{~h}$ MLT. The trace of the footprint for s/c 1 calculated for standard input parameters $\left(p_{\mathrm{dyn}}=2.0 \mathrm{nPa}, D_{s t}=0.0 \mathrm{nT}, B_{y}=0.0 \mathrm{nT}, B_{z}=0.0 \mathrm{nT}\right)$ by the T96 model (Tsyganenko, 1995) is shown in Fig. 1. Also shown are the positions of the CARISMA magnetometer stations and the fields-of-view (FOVs) of two SuperDARN radars located in Saskatoon (looking North-East) and Kapuskasing (looking North-West).

During the hours leading up to the event that will be discussed here, the solar wind conditions were quiet. Between 10:00 and 11:10 UT the ACE satellite measured a steady IMF around $(8,-4,0) \mathrm{nT}$ in GSM coordinates (see Fig. 2). A steady solar wind velocity at $450 \mathrm{~km} / \mathrm{s}$ combined with a particle density of about $6 \mathrm{~cm}^{-3}$ lead to a dynamic pressure of $2.5 \mathrm{nPa}$. A simple time-of-flight analysis suggests that it took the solar wind about 60 min to reach the Earth's magnetopause.

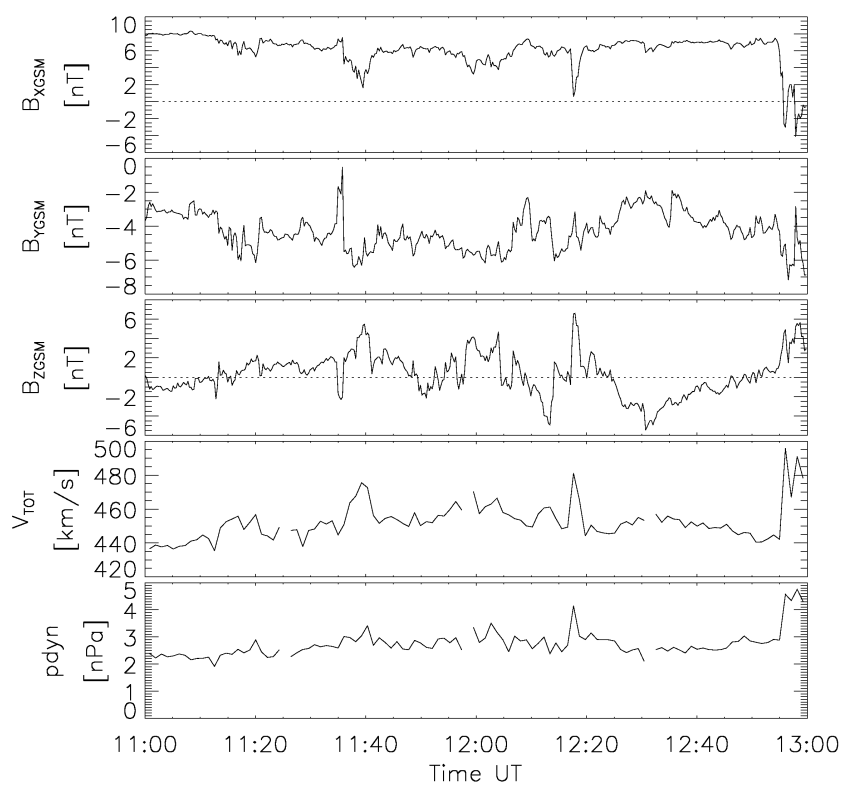

Fig. 2. Overview of the solar wind parameters measured by the ACE satellite.

After 11:10UT the conditions were slightly disturbed, more specifically at 11:35 UT a short $(\sim 1 \mathrm{~min})$ magnetic disturbance together with an increase in dynamic pressure from 2.5 to $3.0 \mathrm{nPa}$ was observed by ACE. The IMF X component increased from 6 to $8 \mathrm{nT}$ and the $\mathrm{Y}$ component shot up from -4 to $-1 \mathrm{nT}$. The $\mathrm{Z}$ component dropped from 2 to $-2 \mathrm{nT}$, leaving the IMF magnitude essentially unchanged at $8 \mathrm{nT}$. This resulted in a clock angle change from $-60^{\circ}$ to $-160^{\circ}$. The disturbance lasted for about one minute before the IMF returned to its original orientation. Lagged by the solar wind propagation time the disturbance is expected to hit the Earth's magnetosphere around 12:35 UT.

Ionospheric velocities measured at 1 min resolution by the two SuperDARN radars located in Saskatoon and Kapuskasing are shown in the top two panels of Fig. 3. The data from Saskatoon's beam 10 and Kapuskasing's beam 5 are shown in the top and second panel, respectively. A pulsation was observed by the Kapuskasing radar between range gate 15 and 30 in all 16 beams of the radar (not shown) after 12:40 UT. It was observed both in E-region scatter at lower range gates and $\mathrm{F}$ region scatter at higher range gates. Just after 12:40 UT it had a peak-to-peak amplitude of about $1000 \mathrm{~m} / \mathrm{s}$ which decreased over the cause of the event. Unfortunately, the data quality is poor, i.e. the data contains a lot of gaps. This makes a Fourier analysis impossible, however from line plots of the velocity the period of the pulsation can be estimated to be between 5 and $6 \mathrm{~min}$.

Although the data quality is bad, the velocities measured by the Kapuskasing radar suggest that the pulsation had a poleward phase propagation, as indicated by the slanted regions of maximum velocity. 
The same pulsation was observed in E region scatter by the Saskatoon radar between range gate 12 to 14 . The data quality here is even worse then at Kapuskasing, however again the period can be estimated to be around $5 \mathrm{~min}$. The areas of the FOVs in which the pulsation was observed are marked dark grey in Fig. 1.

Magnetic field measurements by s/c 1 of Cluster for this event are shown in panels three to five of Fig. 3. The magnetic field is shown in a coordinate system which is aligned along the background magnetic field as predicted by the T96 model.

For each magnetic field datum along the orbit of each satellite the T96 model value gives the background magnetic field direction $\mathcal{F}$. The azimuthal direction component $\mathcal{A}$ is calculated via $\mathcal{A}=\mathcal{F} \times \boldsymbol{r}$ where $\boldsymbol{r}$ is the geocentric position vector of the satellite. Hence the azimuthal unit vector points positive East. The right hand system is completed by a radially inwards pointing component $\mathcal{R}=\mathcal{F} \times \mathcal{A}$.

The advantage of a field-aligned coordinate system is that if a wave is observed, the component in which it is most prominent will indicate its mode (compressional, Alfvénic toroidal or Alfvénic poloidal). The field aligned time series of the magnetic field will contain the compressional mode and the toroidal mode is contained within the azimuthal time series. The poloidal mode will be within the radial direction.

Panels 3, 4 and 5 in Fig. 3 show the radial (poloidal), azimuthal (toroidal) and field-aligned (compressional) magnetic field. The right panels show the respective Fourier powers. As a guide to the eye vertical dashed lines are plotted every five minutes.

The oscillations of interest appear just after 12:35 UT in the compressional component of the magnetic field (fifth panel in Fig. 3). They consist of two cycles and have a period of about five minutes $(3.1 \mathrm{mHz})$. A pulsation with the same frequency, however almost a factor of 4 larger, is observed in the poloidal component (third panel in Fig. 3). A pulsation was also observed in the toroidal component but with a longer period of about seven minutes $(2.4 \mathrm{mHz})$.

The magnetic field measurements in the geographic NorthSouth direction from ground-based magnetometers belonging to the Churchill line of the CARISMA chain are shown in the bottom five panels of Fig. 3. The stations are sorted from highest (TALO) to lowest (PINA) latitude.

At the same time as in the compressional component of s/c 1 all ground-based magnetometer observed a pulsation at $3.1 \mathrm{mHz}$. The latitude profile of the Fourier power and phase at $3.1 \mathrm{mHz}$ of both the North-South (solid line) and East-West (dashed line) component are shown in the left and right panel of Fig. 4, respectively. A maximum in amplitude of the North-South component is observed at the latitude of GILL, along with a $180^{\circ}$ phase shift. No peak is observed in the East-West data. The latitude profiles provide strong evidence that the observed pulsation was a FLR.

Fourier phases at $3.1 \mathrm{mHz}$ along a longitudinal profile of ground based magnetometers allow the determination of the azimuthal wave number $m$. Here we used the data from GILL, RABB and FSMI. Note that FSIM and DAWS (compare Fig. 1) did not observed the pulsation. The average $m$ number is 3.6, as would be expected for such a large scale, externally driven event.

Between 12:10 and 12:35 another pulsation with a frequency of $1.7 \mathrm{mHz}$ was observed by the ground-based magnetometers. Its latitude profile peaked in amplitude around ESKI, accompanied by a roughly $180^{\circ}$ phase shift. The profiles of the East-West component do not show this behaviour, again identifying this pulsation as a FLR.

Note that the pulsation at the lower frequency $(1.7 \mathrm{mHz})$ peaked in amplitude at a higher latitude than that with a higher frequency at $3.1 \mathrm{mHz}$. This behaviour is characteristic of the observation of pulsation events at CMS frequencies (Fenrich et al., 1995).

\subsubsection{Cluster power spectra}

The power spectra of the Cluster magnetic field data presented in Fig. 3 were calculated from the interval for which the time series is shown (12:20 to 13:00 UT). Due to the relatively short interval length, the frequency resolution of these spectra was poor at $0.4 \mathrm{mHz}$.

In order to clearly link the case studies with the statistical analysis presented in the second part of this paper, we already include here a discussion of how Cluster magnetic field data were analysed to produce power spectra which will allow the statistical evaluation of their frequency contents.

Cluster orbit data on 24 October 2004 were searched for the time and position at which each satellite reached perigee. Centred at the time of perigee, the three components of the magnetic field sampled by the four Cluster satellites were selected for an interval with a length of 2500 points. At spin resolution $(4 \mathrm{~s})$ this corresponds to an interval length of $10000 \mathrm{~s}$.

During this interval of about three hours length, each Cluster satellite sampled the magnetic field between $-40^{\circ}$ and $+40^{\circ}$ magnetic latitude. Based on the assumption that the magnetic field is dipolar, this corresponds to $L$ values smaller than $10 R_{E}$. On 24 October 2004 the analysed $10000 \mathrm{~s}$ interval stretched from 10:02 to 12:48 UT which, according to the footprint prediction shown in Fig. 1, maps to latitudes below $75^{\circ}$. It can hence be assumed with confidence that during the entire interval the satellites sampled the conditions on closed magnetic field lines only.

The magnetic field data of the four satellites were converted into the T96 field-aligned coordinate system as described earlier. Subsequently, a linear trend was subtracted from each time series. Spectral powers $\hat{p}\left(f_{i}\right)$ were calculated from the Hanning window tapered time series using a FFT. The interval was $10000 \mathrm{~s}$ long, hence a frequency resolution of $0.1 \mathrm{mHz}$ was achieved. An overall spectrum $p\left(f_{i}\right)$ was then calculated as the mean of the spectral powers of the spacecraft in each frequency bin $f_{i}$. 


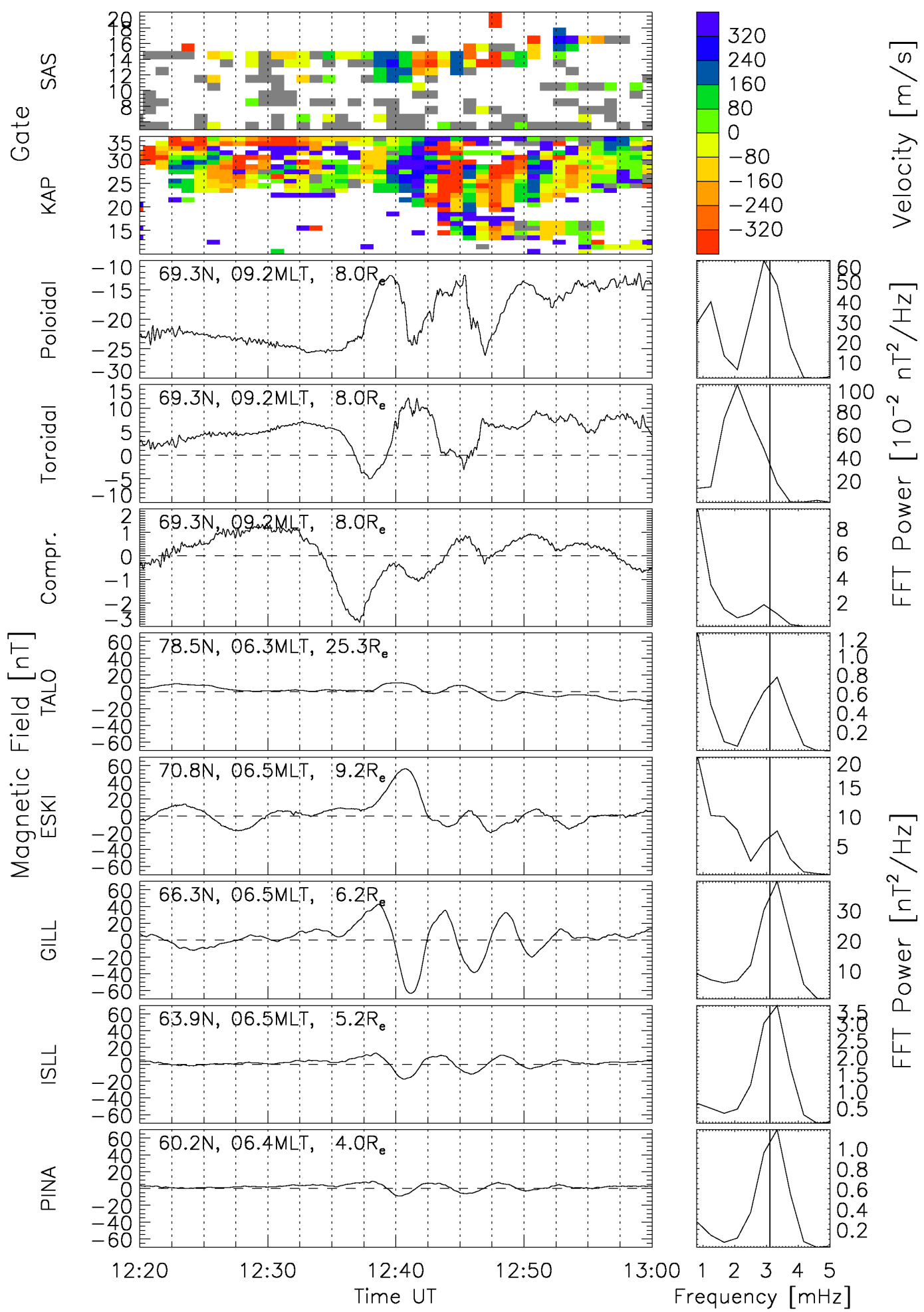

Fig. 3. Ionospheric velocities measured by the Saskatoon and Kapuskasing radar are shown in the top two panels, respectively. The following three panels show magnetic field measurements by s/c 1 in a T96 field-aligned coordinate system. Ground-based magnetometer data from stations along the Churchill line are shown in the bottom five panels. The panels on the right give the respective Fourier spectra. All data are from 24 October 2004. 

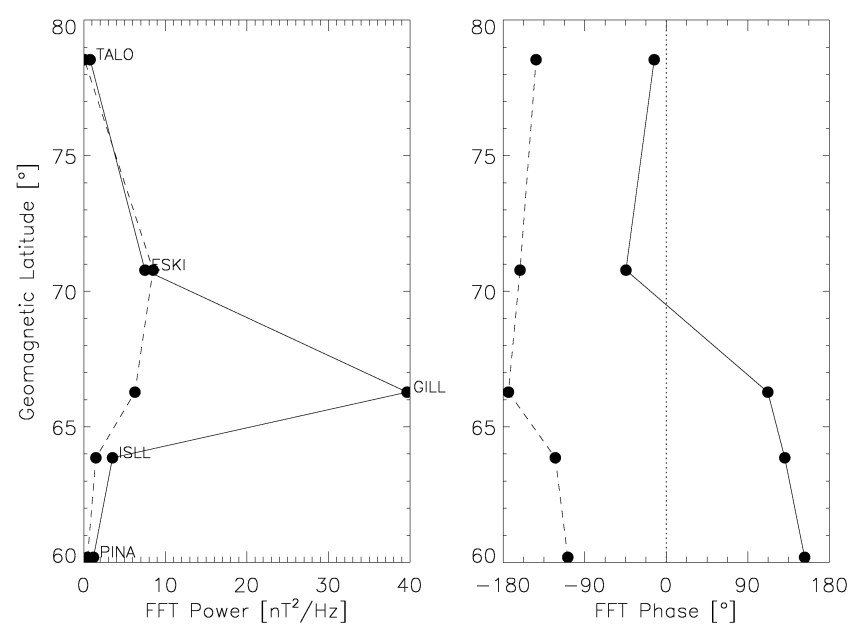

Fig. 4. Latitude profile of Fourier power and phase at $3.1 \mathrm{mHz}$ on 24 October 2004. The profile of the North-South component is shown as solid lines, the dashed lines give the profiles for the East-West component.

Because of the close separation of the Cluster satellites during this event (smaller than $0.5 R_{E}$ ), any "real" wave should appear in data from all four satellites. Taking the average in each frequency bin ensures that artefacts that were only observed by one spacecraft are excluded as much as possible.

To identify peaks in each averaged spectrum, the noise level $P^{\prime}$ was determined between 0.8 and $15.8 \mathrm{mHz}$ in a straight forward way following Walker et al. (1979). First, the average signal power $P$ was determined from the power values $p\left(f_{i}\right)$ as

$P=(1 / n) \sum_{i=0}^{n} p\left(f_{i}\right)$

where $n$ is the number of spectral values between 0.8 and $15.8 \mathrm{mHz}$. An estimate for the noise level $P^{\prime}$ is then obtained by repeating this procedure omitting all points where $p\left(f_{i}\right)>2 P$.

A peak is then defined as a spectral power value that

- is larger than the noise level estimate $P^{\prime}$;

- is larger than at least one neighbouring point both towards higher and lower frequencies.

From all peak values matching the above criteria, those with the highest 20 amplitudes were selected, the others discarded.

To obtain a more accurate estimate of the peak's position, a parabola was fitted to the power value identified as peak and the two adjacent spectral values. The position of the maximum of the fitted parabola was then taken as the peak's position.

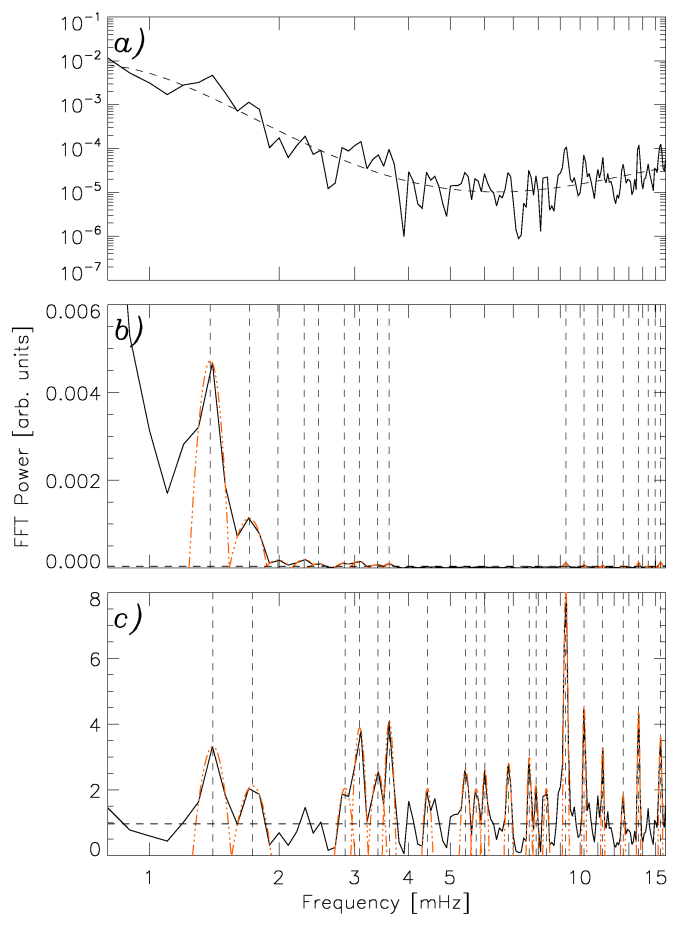

Fig. 5. Panel (a) shows the average spectrum of the compressional magnetic field component for 24 October 2004 in double logarithmic space. The dotted line shows the fitted 4th order polynomial. Panel (b) shows the same spectrum on a linear y-axis. Peaks found in the untreated spectra are marked by vertical dashed lines. See text for further explanation.

Panel (a) in Fig. 5 shows the averaged spectrum of the compressional component for 24 October 2004 in double logarithmic space. The same spectrum but with a linear y axis is shown in panel (b). Vertical dashed lines indicate locations of peaks in the spectrum determined by the algorithm described above. The noise level $P^{\prime}$ is shown as a horizontal dotted line in panel (b) of Fig. 5. Orange dash-dotted lines indicate the parabolas fitted to the peak positions.

In order to whiten the spectra and enhance real peaks, especially at higher frequencies, a 4th order polynomial was fitted in double logarithmic space to the average spectrum and subsequently subtracted from the spectral powers. The dashed line in panel (a) of Fig. 5 shows the polynomial fit. The resulting detrended spectrum is shown in panel (c), in the same format as panel (b) on a linear y-axis.

Figure 5 clearly shows the difference in output when applying the peak-finding procedure to the untreated i.e. undetrended spectrum shown in panel (b) and the treated one in panel (c).

Between 4 and $10 \mathrm{mHz}$ no peaks were detected by the described algorithm in the untreated spectrum, however the treated spectrum clearly shows the existence and relevance of such peaks. Note that in both spectra peaks at 1.7 and $3.1 \mathrm{mHz}$ are detected, as would be expected as Cluster was 


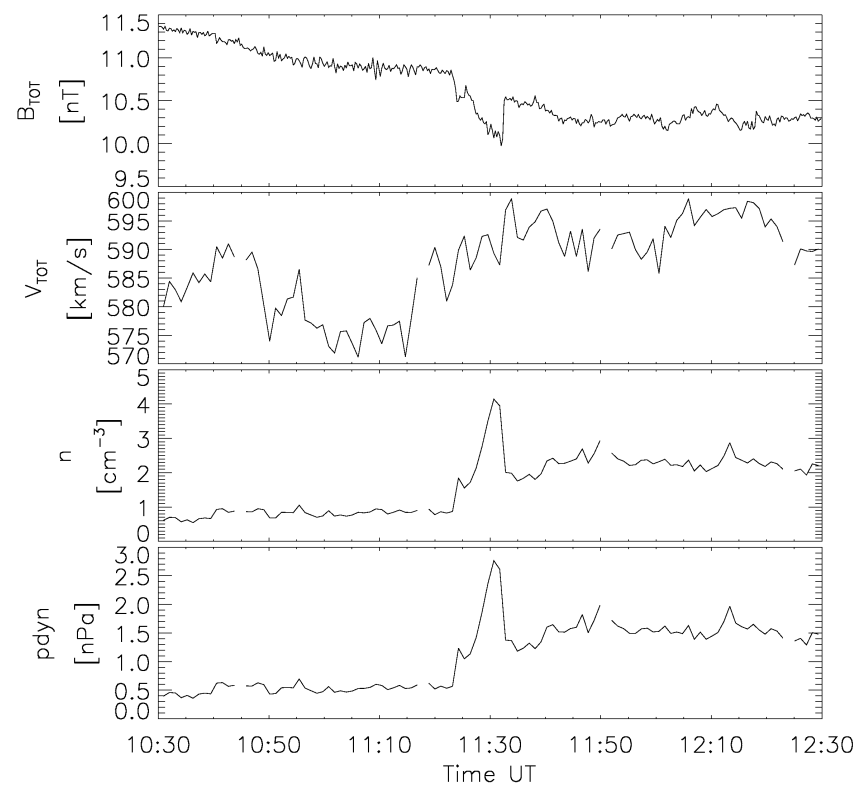

Fig. 6. Unlagged magnetic magnitude and particle data measured by the ACE satellite.

close to the region containing a compressional wave driving the FLRs observed on the ground.

\subsection{November 2004}

The pulsations that were observed on 12 November 2004 were most likely caused by a sudden increase in solar wind pressure. Figure 6 shows the unlagged magnetic magnitude and particle data observed by the ACE satellite. The IMF was steady around $(6,-8,5) \mathrm{nT}$ in GSM coordinates.

During the two hour interval shown in Fig. 6 the solar wind speed was essentially constant at $585 \mathrm{~km} / \mathrm{s}$. Before 11:23 UT the solar wind pressure was constant and low at values around $0.5 \mathrm{nPa}$. After 11:23 UT the solar wind density increased in two steps from 1 to $4 \mathrm{~cm}^{-3}$. Together with a slight increase in solar wind flow speed, this caused a solar wind pressure increase by a factor of 5 . Propagating the pressure pulse at $585 \mathrm{~km} / \mathrm{s}$ predicts that the first smaller pressure increase hit the magnetopause about $50 \mathrm{~min}$ later, i.e. 12:13 UT.

During the time of the event, the Cluster satellites were, according to the T96 model, again magnetically conjugate to the CARISMA stations in Northern America. This is shown in geomagnetic coordinates in Fig. 7 using the same format as Fig. 1. The measurements of the ground- and space-based instruments are shown in Fig. 8 in the same format as Fig. 3.

The magnetometers in Northern America observed a pulsation starting around 12:10 UT, in very good agreement with the predicted arrival time of the pressure pulse. Fourier spectra of the pulsation show that the pulsation consisted of two separated pulsations, one had a frequency of $1.2 \mathrm{mHz}$, the

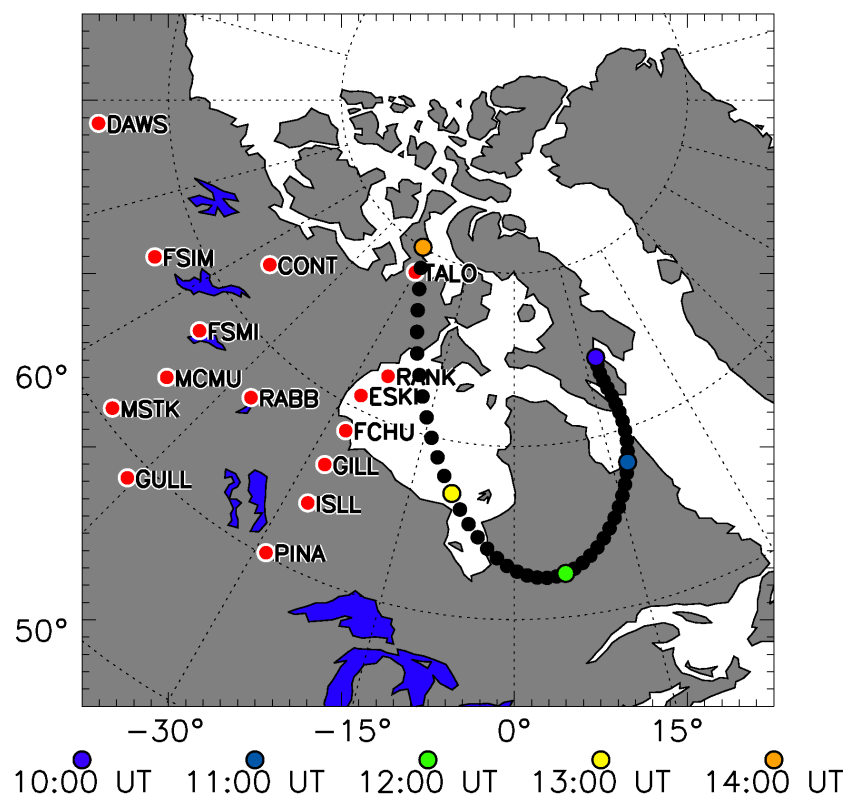

Fig. 7. Locations of the CARISMA magnetometers and the footprint of s/c 1 in geomagnetic coordinates on 12 November 2004.

other oscillated at $2.8 \mathrm{mHz}$. The lower frequency pulsation was the larger one with amplitudes over $200 \mathrm{nT}$ at RANK.

The pulsation was also observed by the four Cluster satellites. The unfiltered magnetic field data in the T96 fieldaligned system is shown in the top three panels of Fig. 8 . The variation causes the spectral peak at $1.2 \mathrm{mHz}$ which can be clearly observed in the spectra on the right. Although not immediately obvious from the time series data, the Fourier spectrum of the compressional component reveals that wave power at the second frequency at $2.8 \mathrm{mHz}$ was observed by the satellites as well. The poloidal components of the magnetic field measured by Cluster shows some sign of the pulsation near $1.2 \mathrm{mHz}$.

Latitude profiles of the ground-based magnetometer data for the two dominant frequencies are shown in Fig. 9. The latitude profile at $1.2 \mathrm{mHz}$ is shown in the top panel, that at $2.8 \mathrm{mHz}$ is shown in the bottom. Both show the characteristic peak at one latitude with a $180^{\circ}$ phase shift across that latitude. As would be expected, the lower frequency peaked at higher latitudes, i.e. on longer field lines.

Applying the same scheme as described in Sect. 2.1.1 to the magnetic field data of the Cluster satellites during this interval again extracts, among others, the two frequencies at which FLRs were observed on the ground.

We have presented two events where pulsation activity was observed in the compressional component by the Cluster satellites simultaneously to FLR activity on the ground. It was shown that our algorithm detected peaks at the FLR frequency in the spectra of the compressional magnetic field component measured by Cluster. In both cases we propose that the signatures measured by the Cluster satellites are 

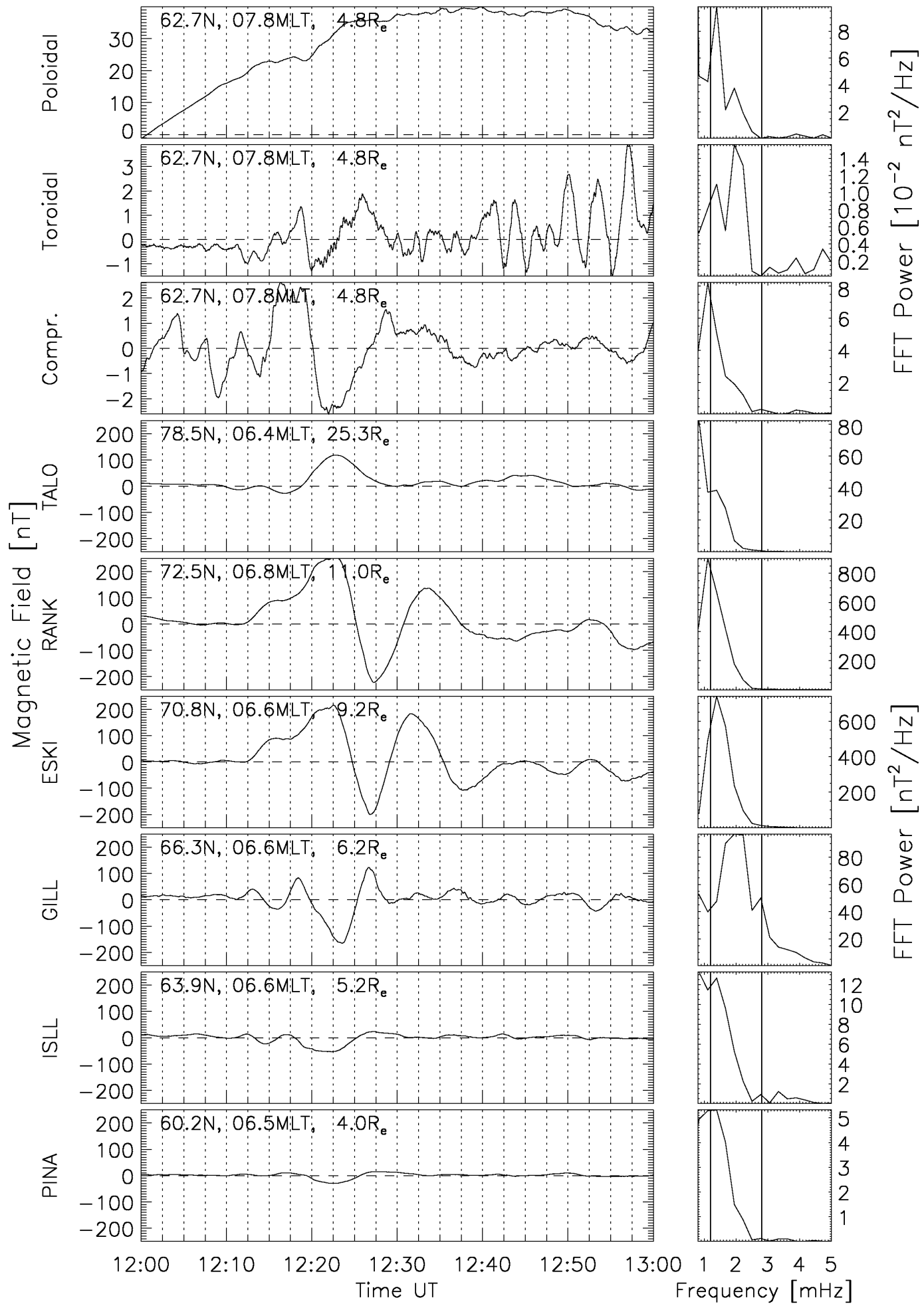

Fig. 8. The top three panels show magnetic field measurements by s/c 4 in the T96 field-aligned coordinate system. Ground-based magnetometer data from stations along a latitude profile of CARISMA stations are shown in the bottom seven panels. The panels on the right give the respective Fourier spectra. All data are from 12 November 2004. 

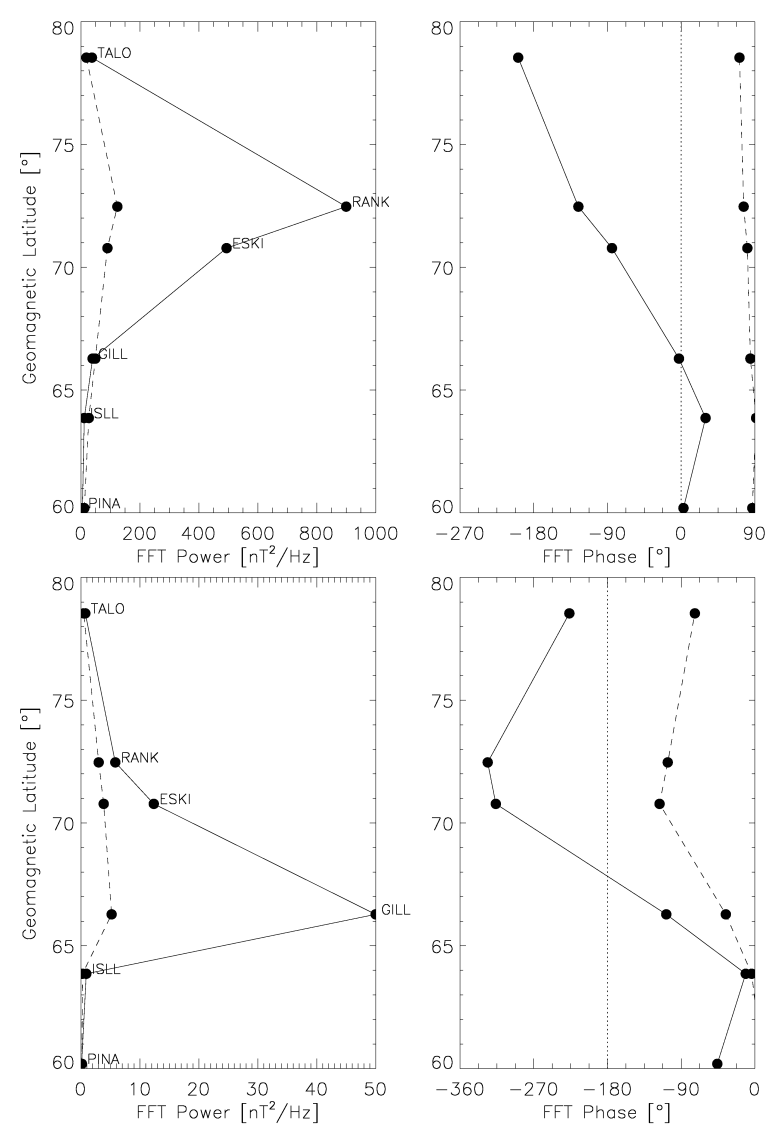

Fig. 9. Latitude profile of Fourier power and phase at $1.2 \mathrm{mHz}$ (top panels) and $2.8 \mathrm{mHz}$ (bottom panels) for 12 November 2004.

waveguide/cavity modes that drive the FLR which is then observed on the ground.

Based on this assumption, in the following section we go on to search for peaks in magnetic field data gathered by Cluster and investigate whether those favour a certain set of frequencies.

\section{Statistical analysis}

\subsection{Data preparation}

Between June 2004 and June 2005 the orbits of the four Cluster satellites did not change significantly. Hence their respective separation stayed essentially constant while they sampled the plasma conditions at all MLT. The maximum separation at the perigee leg of the orbit was $0.8 R_{E}$ between $\mathrm{s} / \mathrm{c} 1$ and s/c 3 , whereas s/c 1 and s/c 4 were only separated by $0.1 R_{E}$. The average distance between the spacecraft during a typical orbit around perigee was $0.4 R_{E}$.

For each of the 154 orbits between 1 June 2004 and 31 May 2005 the time and position at which each Cluster satellite crossed the perigee was determined. The three component time series of the four spacecraft were visually inspected

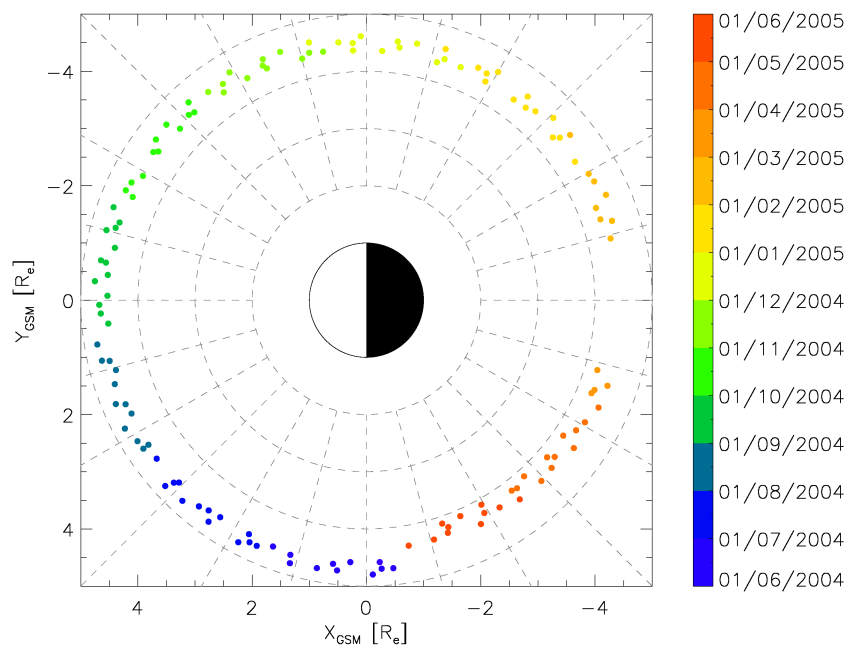

Fig. 10. The MLT position of the perigee and the minimum geocentric distance of all the Cluster passes analysed in this study. The colour of each datum gives the month according to the colour bar on the right.

to spot obvious problems with the data. Unfortunately, all orbits between 23:00 and 01:00 MLT had to be rejected due to large data gaps of the order of half an hour during the perigee pass. These data gaps were caused by Cluster spending the time around perigee in the shadow of the Earth, hence no sun-pulse was available to determine the correct spin rate, making the data calibration impossible. All in all, of the 154 passes 20 were rejected for s/c 1,23 for s/c 2, 22 for s/c 3 and 25 for s/c 4 . This inspection left 132 orbits for the statistical study.

The perigee positions and their respective times of the orbits which were used in this study are shown in Fig. 10. The colour of the points give the month in which the Cluster satellites completed the marked orbit and hence provide a relation between time and MLT. All orbits had closest approaches between 4 and $5 R_{E}$ when projected into the X-Y GSM plane.

Centred at the time of perigee, time series of the magnetic field data of $10000 \mathrm{~s}$ length were transformed into the T96 field-aligned coordinate system for all 132 orbits. Then, a FFT was calculated of each time series and the power values were averaged in each frequency bin over the four satellites to generate a resulting spectrum $p\left(f_{i}\right)$. The spectrum was subsequently whitened by subtracting a fitted 4 th order polynomial.

Peaks in each whitened spectrum were then identified using the criteria outlined in Sect. 2.1.1. We end up with a list of intervals, the MLT of the perigee during that interval and a list of frequencies at which the overall spectrum showed peaks.

In the following section we will investigate whether our data contains evidence that spectral peaks in the magnetic field tend to occur preferentially at a certain set of frequencies, including the CMS frequencies. 


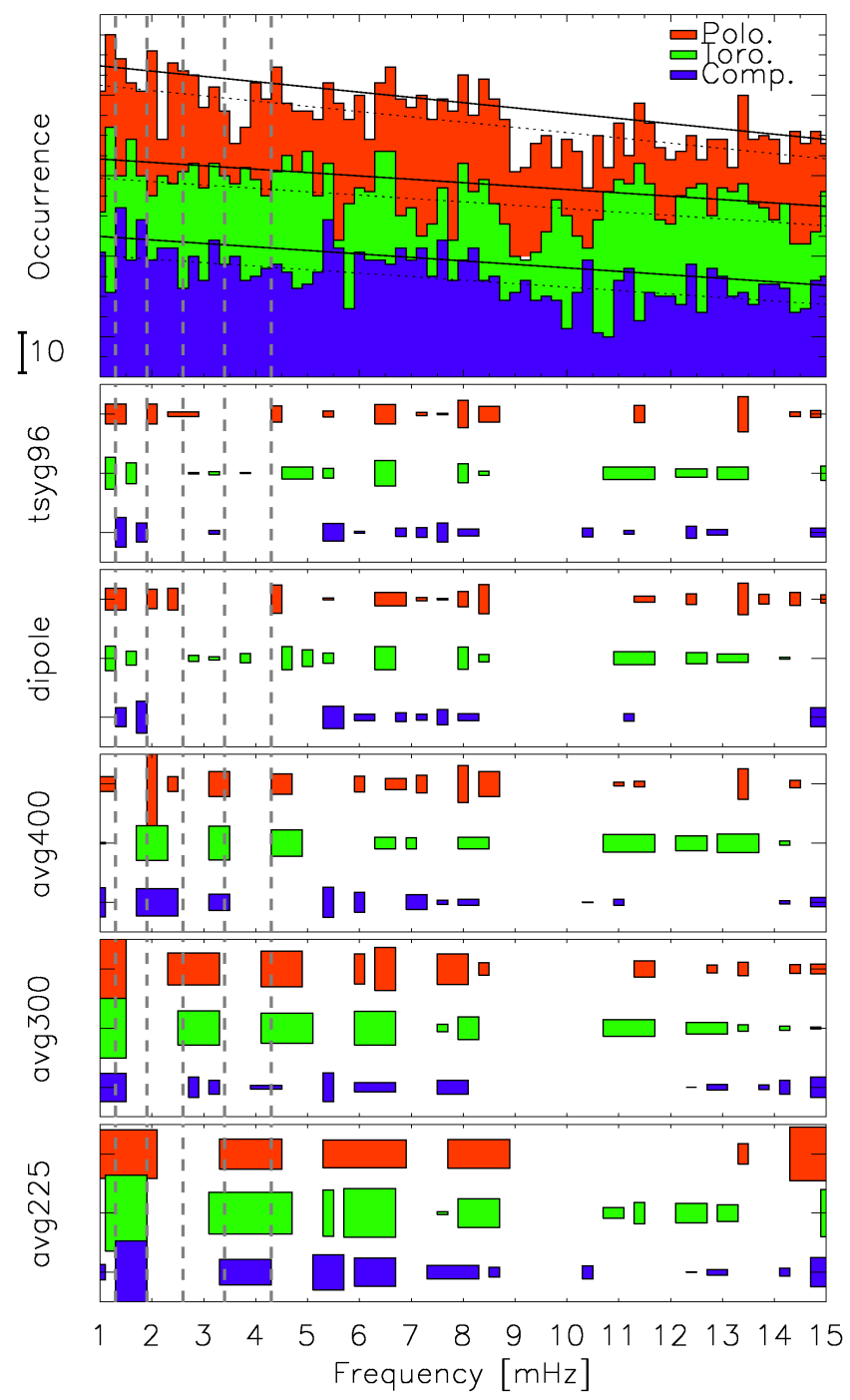

Fig. 11. Occurrence of spectral peaks in the three magnetic field components when using the T96 model (top panel). The dashed lines give the linear fit to each histogram, the solid lines shows the 95\% confidence level of each fit. The second panel shows the significant peaks of each histogram. Subsequent panels show characteristics of significant peaks in occurrence distributions determined from different magnetic field models. See text for further details.

\subsection{Statistical results}

The number of peaks in $0.2 \mathrm{mHz}$ frequency bins detected in each of the three magnetic field components are shown in the top panel of Fig. 11. The occurrence rates of the three components have been offset in order to facilitate a comparison of peak positions in the three histograms. The CMS frequencies are marked by grey vertical dashed lines.

In Fig. 11 the dotted lines give a linear fit to the occurrences in each component, the solid lines give the $95 \%$ confidence level of each fit. Any occurrence above the $95 \%$ confidence level is marked as a significant peak in the distribution.
The second panel in Fig. 11 shows the position of significant peaks in the three data sets. The markers are scaled in the $y$ direction according to the level of each peak above the 95\% confidence line. The $\mathrm{x}$ direction is scaled according to the width of the peak.

We find some indications that pulsations preferentially occur at CMS frequencies in all three magnetic field components. However, none of the three data sets shows a preference for all CMS frequencies. The preference is also not as pronounced as was found in studies confirming the existence of CMS frequency preference (Samson et al., 1991; Fenrich et al., 1995; Provan and Yeoman, 1997; Mathie et al., 1999; Plaschke et al., 2009).

The poloidal and toroidal data sets mostly show preferred frequencies, i.e. peaks, at the same frequencies. The peaks in the compressional component are found at somewhat different locations.

To investigate the influence of the magnetic field model on the peak occurrence, the same statistical study was repeated using a dipolar magnetic field to predict the field-aligned direction. The resulting occurrence histogram is not shown, however the positions and amplitudes of the significant peaks in the distribution are shown in the third panel in Fig. 11. The positions and amplitudes of the significant peaks differ very little from those presented in the second panel. This is to be expected as the Cluster satellites spend a large part of the orbit around perigee in the inner magnetosphere where the T96 model is essentially dipolar.

We repeated the analysis using an average over a variety of actual magnetic field measurements by the Cluster satellites to determine the background magnetic field direction. As for the dipole magnetic field, the characteristics of the significant peaks found in the occurrence histograms are shown in the bottom three panels of Fig. 11.

The number of data points from which the average was calculated to determine the background magnetic field direction were chosen to be $400(27 \mathrm{~min}), 300(20 \mathrm{~min})$ and $225(15 \mathrm{~min})$ and the results are shown in the fourth, fifth and sixth panel of Fig. 11, respectively. Again, evidence for a preference of certain CMS frequencies is found, however which specific frequency is preferred depends on the average length.

We find that the position of significant peaks below $5 \mathrm{mHz}$ varies considerably with the magnetic field model. Above $5 \mathrm{mHz}$, however, we find consistent peaks independent of the magnetic field model used. Specifically, we find peaks in the poloidal component around $6.5,8,11.5$ and $13.5 \mathrm{mHz}$. The toroidal component shows reappearing peaks at $6.5,8$, $11,12.5$ and $13 \mathrm{mHz}$. Consistent peaks in the compressional component are found at 5.5, 7.5, and $15 \mathrm{mHz}$.

We hypothesise that a preference for the stated frequencies above $5 \mathrm{mHz}$ is caused by the same mechanism as was put forward to explain CMS frequencies. The harmonic series of the cavity/waveguide is being detected in the region close to where it drives FLRs. However, as will be discussed in the 
next section, the polar orbit of the Cluster spacecraft favours the analysis of frequencies higher than the CMS frequencies.

The peak data provided by the scheme described in Sect. 2.1.1 has also been binned in many categories that might effect the distribution of peak occurrence. It is for example well established that the solar wind conditions and the local time have a dominating influence on the observation of Pc5 pulsations within the magnetosphere. Hence the results were binned according to solar wind conditions and position of orbit in MLT, however the resulting histograms did not alter significantly from those presented in Fig. 11.

\subsection{A note on the Cluster orbit}

Some characteristics of a typical Cluster orbit are shown in Fig. 12. The Cluster satellites typically sample magnetic field lines with L-values between 6 and 10 only at the beginning and end of each interval; the middle $60 \%$ of the interval they spend on field lines with $\mathrm{L}$ values smaller than $6 R_{E}$.

FLRs in the Pc5 frequency range, specifically at CMS frequencies, tend to occur at magnetic latitudes above $60^{\circ}$ on field lines with $\mathrm{L}$ values between 6 and $10 R_{E}$. In the cavity/waveguide model, the turning point of the driving compressional wave lies at larger $\mathrm{L}$ values than the resonance region. Toward smaller $\mathrm{L}$ values the compressional driver decays exponentially. Hence the Cluster orbit is not ideal for the study of cavity/waveguide modes at CMS frequencies as it crosses the inner magnetosphere earthwards of the expected turning point.

When the satellites sample field lines having $L$ values between 6 and $10 R_{E}$, i.e. on field lines which can support FLRs at CMS frequencies, they are generally located at magnetic latitudes between 20 and $40^{\circ}$. Hence they are a significant distance ( $3 R_{E}$ or so) away from the magnetic equatorial plane. The equatorial plane is the region where one would intuitively expect the cavity/waveguide modes to form. This preference for the equatorial plane has been suggested by modelling studies by Lee and Lysak (1989).

However, the top panel of Plate 3 in Lee and Lysak (1989) shows the leakage of significant compressional power to magnetic latitude above $20^{\circ}$. Hence signatures of cavity/waveguide modes at CMS frequencies can be expected to be observed by the Cluster satellites. Furthermore, the two case studies presented here show that Cluster observed significant compressional wave power during FLR occurrences even though it was located inside the evanescent region of the cavity/waveguide mode.

\section{Conclusions}

We have described two case studies during which groundbased magnetometers and HF radars detected FLRs in the Pc5 frequency range, specifically at CMS frequencies. A careful analysis of power spectra compiled from an extended $10000 \mathrm{~s}$ interval during which the Cluster satellites

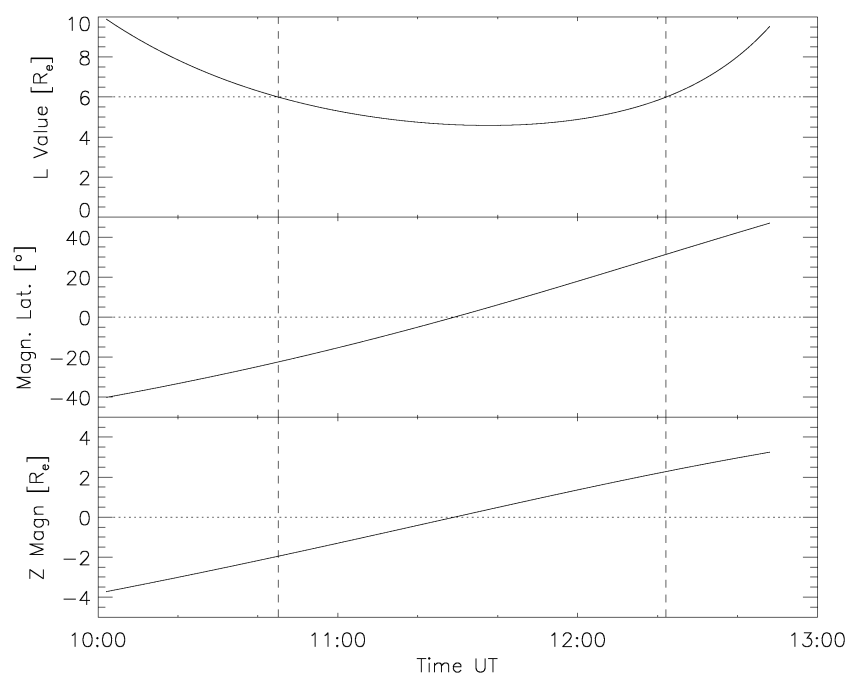

Fig. 12. Characteristics of a typical Cluster orbit. For 24 October 2004 the L value (top panel), magnetic latitude (middle panel) and distance from the magnetic equator (bottom panel) are shown.

were magnetically conjugate to the resonance region exhibits peaks at the FLR frequency in both cases. Most importantly, peaks at the FLR frequencies were found in the spectra of the compressional component, indicating that signatures of the cavity/waveguide mode driving the FLR were picked up by Cluster during both events.

Following on from the two case studies where compressional power in the Cluster magnetic field measurements could be connected to FLR activity detected on the ground we expanded the analysis of such $10000 \mathrm{~s}$ intervals to one year's worth of Cluster data. An algorithm was use to automatically detect peaks in spectra of the compressional, poloidal and toroidal magnetic field component of 132 such orbits between May 2004 and June 2005.

We presented histograms of the occurrence of spectral peaks in $0.2 \mathrm{mHz}$ wide frequency bins between 1 and $15 \mathrm{mHz}$ and peaks in the occurrence rate are identified. Although previous studies have reported that FLRs occur with a stable reoccurring set of frequencies we find no consistent preference for all CMS frequencies in our data.

Repeating the statistical analysis using 5 different magnetic field models we again find no consistent preference for the CMS frequencies. We do find, however, indications for a preference of certain frequencies above $5 \mathrm{mHz}$, depending on the magnetic field component. We hypothesise that the mechanism selecting these frequencies is the same waveguide/cavity model as was put forward to explain the CMS frequencies. However, in the inner magnetosphere, where Cluster's perigee is located, we measure higher harmonics of the cavity/waveguide than the CMS frequencies.

The fact that no clear preference for pulsations occurring at CMS frequencies could be found in the presented data set might be due to the spacecraft's orbit. The Cluster perigee is 
located significantly inwards of the expected position of the turning point of the cavity/waveguide mode driving a FLR in the Pc5 range. Using data from a spacecraft with a different orbit configuration, specifically Themis, can circumvent this problem and might potentially show different results.

Acknowledgements. The authors would like to thank E. A. Lucek and the rest of the Cluster FGM team for maintaining the instruments as well as the Cluster Active Archive for making the data available. L. B. N. Clausen acknowledges funding from the European Commission under the Marie Curie Host Fellowship for Early Stage Research Training SPARTAN, Contract No MEST-CT-2004007512, University of Leicester, UK.

Topical Editor I. A. Daglis thanks P. Francia and another anonymous referee for their help in evaluating this paper.

\section{References}

Baker, G. J., Donovan, E. F., and Jackel, B. J.: A comprehensive survey of auroral latitude Pc5 pulsation characteristics, J. Geophys. Res., 108, 1384, doi:10.1029/2002JA009801, 2003.

Chen, L. and Hasegawa, A.: A theory of long-period magnetic pulsations, 1 . Steady state excitation of field line resonance, J. Geophys. Res., 79, 1024-1032, 1974.

Clausen, L. B. N., Yeoman, T. K., Fear, R. C., Behlke, R., Lucek, E. A., and Engebretson, M. J.: First simultaneous measurements of waves generated at the bow shock in the solar wind, the magnetosphere and on the ground, Ann. Geophys., 27, 357-371, 2009, http://www.ann-geophys.net/27/357/2009/.

Dungey, J. W.: Electrodynamics of the outer atmosphere, in: Ionos. Res. Sci. Rep. 69, Pennsylvania State University, 1954.

Engebretson, M. J., Zanetti, L. J., Potemra, T. A., and Acuna, M. H.: Harmonically structured ULF pulsations observed by the AMPTE CCE magnetic field experiment, Geophys. Res. Lett., 13, 905-908, 1986.

Eriksson, P. T. I., Walker, A. D. M., and Stephenson, J. A. E.: A statistical correlation of Pc5 pulsations and solar wind pressure oscillations, Adv. Space Res., 38, 1763-1771, doi:10.1016/j.asr. 2005.08.023, 2006.

Fenrich, F. R., Samson, J. C., Sofko, G., and Greenwald, R. A.: ULF high- and low-m field line resonances observed with the Super Dual Auroral Radar Network, J. Geophys. Res., 100, 2153521548, doi:10.1029/95JA02024, 1995.

Jacobs, J. A., Kato, Y., Matsushita, S., and Troitskaya, V. A.: Classification of Geomagnetic Micropulsations, J. Geophys. Res., 69, 180, doi:10.1029/JZ069i001p00180, 1964.

Kivelson, M. G. and Southwood, D. J.: Resonant ULF waves - A new interpretation, Geophys. Res. Lett., 12, 49-52, 1985.

Kivelson, M. G. and Southwood, D. J.: Coupling of global magnetospheric MHD eigenmodes to field line resonances, J. Geophys. Res., 91, 4345-4351, 1986.

Kivelson, M. G., Etcheto, J., and Trotignon, J. G.: Global compressional oscillations of the terrestrial magnetosphere: the evidence and a model., J. Geophys. Res., 89, 9851-9856, 1984.

Lee, D.-H. and Lysak, R. L.: Magnetospheric ULF wave coupling in the dipole model - The impulsive excitation, J. Geophys. Res., 94, 17097-17103, 1989.

Mathie, R. A., Mann, I. R., Menk, F. W., and Orr, D.: Pc5 ULF pulsations associated with waveguide modes observed with the
IMAGE magnetometer array, J. Geophys. Res., 104, 7025-7036, doi:10.1029/1998JA900150, 1999.

Plaschke, F., Glassmeier, K.-H., Auster, H. U., Constantinescu, O. D., Magnes, W., Angelopoulos, V., Sibeck, D. G., and McFadden, J. P.: Standing Alfvén waves at the magnetopause, Geophys. Res. Lett., 36, 2104, doi:10.1029/2008GL036411, 2009.

Prikryl, P., Greenwald, R. A., Sofko, G. J., Villain, J. P., Ziesolleck, C. W. S., and Friis-Christensen, E.: Solar-wind-driven pulsed magnetic reconnection at the dayside magnetopause, Pc5 compressional oscillations, and field line resonances, J. Geophys. Res., 103, 17307-17322, doi:10.1029/97JA03595, 1998.

Provan, G. and Yeoman, T. K.: A comparison of field-line resonances observed at the Goose Bay and Wick radars, Ann. Geophys., 15, 231-235, 1997, http://www.ann-geophys.net/15/231/1997/.

Rostoker, G., Akasofu, S.-I., Foster, J., Greenwald, R. A., Lui, A. T. Y., Kamide, Y., Kawasaki, K., McPherron, R. L., and Russell, C. T.: Magnetospheric substorms - Definition and signatures, J. Geophys. Res., 85, 1663-1668, doi:10.1029/ JA085iA04p01663, 1980.

Samson, J. C. and Rostoker, G.: Latitude-dependent characteristics of high-latitude Pc 4 and Pc 5 micropulsations, J. Geophys. Res., 77, 6133-6144, 1972.

Samson, J. C., Jacobs, J. A., and Rostoker, G.: Latitude-Dependent Characteristics of Long-Period Geomagnetic Micropulsations, J. Geophys. Res., 76, 3675-3683, doi:10.1029/JA076i016p03675, 1971.

Samson, J. C., Greenwald, R. A., Ruohoniemi, J. M., Hughes, T. J., and Wallis, D. D.: Magnetometer and radar observations of magnetohydrodynamic cavity modes in the earth's magnetosphere, Can. J. Phys., 69, 929-937, 1991.

Samson, J. C., Harrold, B. G., Ruohoniemi, J. M., Greenwald, R. A., and Walker, A. D. M.: Field line resonances associated with MHD waveguides in the magnetosphere, Geophys. Res. Lett., 19, 441-444, 1992.

Samson, J. C., Waters, C. L., Menk, F. W., and Fraser, B. J.: Fine structure in the spectra of low latitude field line resonances, Geophys. Res. Lett., 22, 2111-2114, doi:10.1029/95GL01770, 1995.

Southwood, D. J.: Some features of field line resonances in the magnetosphere, Planet. Space Sci., 22, 483-491, 1974.

Stephenson, J. A. E. and Walker, A. D. M.: HF radar observations of Pc5 ULF pulsations driven by the solar wind, Geophys. Res. Lett., 29, 090 000-1, doi:10.1029/2001GL014291, 2002.

Takahashi, K.: ULF waves: 1997 IAGA division 3 reporter review, Ann. Geophys., 16, 787-803, 1998, http://www.ann-geophys.net/16/787/1998/.

Tsyganenko, N. A.: Modeling the Earth's magnetospheric magnetic field confined within a realistic magnetopause, J. Geophys. Res., 100, 5599-5612, 1995.

Viall, N. M., Kepko, L., and Spence, H. E.: Relative occurrence rates and connection of discrete frequency oscillations in the solar wind density and dayside magnetosphere, J. Geophys. Res., 114, 1201, doi:10.1029/2008JA013334, 2009.

Walker, A. D. M., Greenwald, R. A., Stuart, W. F., and Green, C. A.: STARE auroral radar observations of Pc 5 geomagnetic pulsations, J. Geophys. Res., 84, 3373-3388, 1979.

Waters, C. L., Takahashi, K., Lee, D.-H., and Anderson, B. J.: Detection of ultralow-frequency cavity modes using spacecraft data, J. Geophys. Res., 107, 1284, doi:10.1029/2001JA000224, 2002. 